\title{
BMJ Open Past and recent abuse is associated with early cessation of breast feeding: results from a large prospective cohort in Norway
}

Marie Flem Sørbø, ${ }^{1,2}$ Mirjam Lukasse, ${ }^{1,3}$ Anne-Lise Brantsæter, ${ }^{4}$ Hilde Grimstad ${ }^{1}$

To cite: Sørbø MF,

Lukasse M, Brantsæter A-L, et al. Past and recent abuse is associated with early cessation of breast feeding: results from a large prospective cohort in Norway. BMJ Open 2015:5:e009240. doi:10.1136/bmjopen-2015009240

- Prepublication history and additional material is available. To view please visit the journal (http://dx.doi.org/ 10.1136/bmjopen-2015009240).

Received 29 June 2015 Revised 14 October 2015 Accepted 19 October 2015

CrossMark

For numbered affiliations see end of article.

Correspondence to

Marie Flem Sørbø;

marie.flem.sorbo@ntnu.no

\section{ABSTRACT}

Objective: Breast feeding provides a wide range of health benefits for both infants and mothers. Few studies have examined the impact of past and recent abuse of women on breastfeeding behaviour. The aims of our study were to examine whether exposure to past and recent emotional, sexual or physical abuse was associated with early breastfeeding cessation, and to assess whether a potential association differed for known and unknown perpetrators.

Design: Prospective cohort study.

Setting: Norway, years 1999-2006.

Participants: 53934 mothers participated in the Norwegian Mother and Child Cohort Study. We included mothers with singleton pregnancy who had responded to three questionnaires (weeks 18 and 30 in pregnancy, and 6 months postpartum) and had answered minimum one of the abuse questions in week 30 .

Main outcome measure: ORs were estimated by binary logistic regression with cessation of any (all) breast feeding before 4 months as the outcome, and abuse including subcategories of abuse, as the exposure.

Results: Nearly all women initiated breast feeding, but $12.1 \%$ ceased any breast feeding before 4 months and $38.9 \%$ ceased full breast feeding before 4 months, but continued partial breast feeding. Overall, $19 \%$ of the women reported any adult abuse and $18 \%$ reported any child abuse. The highest risk of any breast feeding cessation before 4 months was seen in women exposed to three types of adult abuse (emotional, sexual or physical), with adjusted OR being $1.47(95 \% \mathrm{Cl} 1.23$ to 1.76) compared with no abuse. Recent abuse and exposure from known perpetrator resulted in nearly $40 \%$ and $30 \%$ increased risk, respectively. The OR of any breast feeding cessation for women exposed to any child abuse was 1.41 (95\% Cl 1.32 to 1.50) compared with no abuse in childhood.

Conclusions: Past and recent abuse of women is strongly associated with early cessation of breast feeding. Abused mothers comprise a key group to target for extra support and breastfeeding assistance.

\section{BACKGROUND}

Breast feeding has long been acknowledged as the optimal infant nutrition conferring

\section{Strengths and limitations of this study}

- This is one of the largest studies to report an association between abuse of women and increased risk of early breastfeeding cessation. Our study provides new significant information about singular and combined types of abuse and breast feeding (emotional, sexual and physical abuse) from one of the largest prospective population-based pregnancy cohorts. It is an important contribution to the field because it is one of the first to document the association between emotional abuse, as singular and combined groups, and breastfeeding cessation.

- The increased risk of early breastfeeding cessation among women who have experienced abuse was observed independently of prior depression, postpartum depression, and other potential confounders and intermediate factors.

- Major strengths of this study include the prospective design; the large sample of women from all regions of Norway, including all age and socioeconomic groups; and detailed information about experiences of abuse, including type of abuse, whether past or recent abuse, and potential confounding factors.

- Since this study is observational, no causal implications can be drawn, and although confounding by other variables was carefully considered, residual confounding cannot be excluded.

beneficial short-term and long-term health effects for both infants and mothers. ${ }^{1-5}$ The WHO has since 2001 recommended exclusive breast feeding for the first 6 months of life ${ }^{6-8}$ and this has been is adopted by the Norwegian Health Authorities. ${ }^{9}$

Abuse of women is common worldwide, as one in three women during lifetime suffer partner or non-partner abuse. ${ }^{10}$ There is an increasing body of research showing that recent and past abusive experiences influences women's physical and mental health negatively, and have a negative impact on reproductive and postpartum health. ${ }^{11}$ Adverse effects include adolescent and 
unintended pregnancies, miscarriages, sexually transmitted diseases and postpartum depression, ${ }^{11-14}$ all of which are barriers to breast feeding.

Given the overwhelming evidence of the positive effects of breast feeding, knowledge about factors influencing breastfeeding behaviour is essential. Norway has one of the highest breastfeeding rates in the world and almost all mothers initiate breast feeding. ${ }^{4}{ }^{15} 16$ Factors that positively influence breast feeding in Norway are higher educational level of the mother, higher maternal age, being married and multiparity; however, smoking and obesity have shown a negative impact. ${ }^{15} 1718$ There is a lack of knowledge about the impact of past and recent abuse of women on breastfeeding behaviour. Only a few studies have been published and these are preliminary and inconclusive. Most studies have examined only one type of abuse, that is, sexual or physical, although these often occur simultaneously and studies that included perpetrators mostly focused on abuse from partner only. ${ }^{19-23}$ The impact of emotional abuse on breast feeding has rarely been studied. ${ }^{20}{ }^{23}$ Furthermore, studies are difficult to compare due to different designs and various definitions of both abuse and breast feeding. The samples are often small, based on clinical cohorts and with a cross-sectional design, hence not applicable to the broader population.

We explored the impact of abuse of women on breastfeeding behaviour in a large prospective population in Norway where the expectations to breast feed are high, and breast feeding is facilitated in the work regulations, for example, paid leave for 1 year. The first aim of our study was to examine whether exposure to adult emotional, sexual or physical abuse as a singular or combined exposure was associated with early breastfeeding cessation. Second, we wanted to assess whether a potential association differed for adult recent and non-recent abuse, and for known and unknown perpetrators. Third, we wanted to examine the association between child abuse and early breastfeeding cessation.

\section{METHODS}

\section{Population and study design}

The Norwegian Mother and Child Cohort Study $(\mathrm{MoBa})$ is a prospective population-based pregnancy cohort conducted by the Norwegian Institute of Public Health. ${ }^{24}$ The participants were recruited to the study through a postal invitation in connection with a routine ultrasound examination offered to all pregnant women in Norway. Participants were recruited from all over Norway from 1999 to 2008 , and $40.6 \%$ of invited women consented to participate. The cohort now includes 114500 children and 95200 mothers. The women were asked to answer questionnaires at regular intervals during pregnancy and after birth. In the current study, we used information from three questionnaires: the baseline questionnaire completed around week 18 of pregnancy (sociodemographics and risk factors), the questionnaire answered in gestational week 30 (abuse questions), and the first follow-up questionnaire after delivery (breastfeeding questions), completed at infant aged 6 months (questionnaires available at http://www. fhi.no/moba). MoBa files are linked to pregnancy and birth records from the Norwegian Medical Birth Registry (NMBR). Written informed consent was obtained. The current study is based on version IV of the quality-assured data files, including participants recruited in years 1999-2006. The research was performed in accordance with the Strobe guidelines. ${ }^{25} \mathrm{An}$ outline of the Strobe guidelines is added in the online supplementary material.

The inclusion of the study population is described in figure 1 . The source population study comprised women who had filled in all three questionnaires and were registered in NMBR $(n=64714)$. For women participating with more than one pregnancy, only information from the first pregnancy was included. Furthermore, we only included women with singleton pregnancies and those who had answered a minimum of one of the abuse questions, leaving a total of 53934 for descriptive characteristics. For all adjusted analyses, we included only women with complete information on the exposures and covariates.

\section{VARIABLES}

\section{Exposure variables-different abuse categories}

The abuse questions and response options are shown in online supplemental figure S1. These questions were part of the third MoBa questionnaire, which was responded to at gestational week 30 . The two questions about emotional abuse are similar to those in the

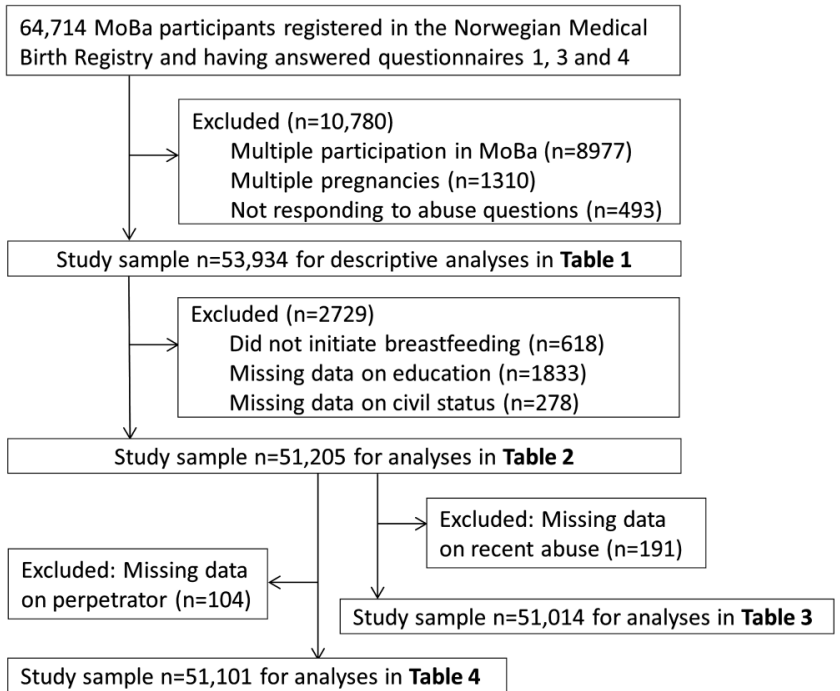

Figure 1 Flow chart of inclusion. Questionnaire 1 was answered in gestational week 18, questionnaire 3 in gestational week 30 and questionnaire 4 was answered 6 months postpartum (MoBa, Norwegian Mother and Child Cohort Study). 
Norvold Abuse Questionnaire, ${ }^{26}$ which measures mild and severe emotional abuse. We merged the responses to the two emotional abuse questions into one variable. Women could respond 'no never' to the various types of abuse or 'yes' as an adult ( $\geq 18$ years) and/or as a child ( $<18$ years) to the various types of abuse. The question about sexual abuse with response options is a modified version of the sexual abuse question in the Abuse Assessment Screen. ${ }^{27}$ This screening tool is not validated, but has been used in other studies. ${ }^{28} 29$ The question about physical abuse has been used in other studies, but is not validated. ${ }^{30}{ }^{31}$ Women who answered 'yes' to at least one of the adult abuse questions, that is, past or recent adult abuse, were defined as having suffered from any adult abuse. Likewise, women responding 'yes' to one or more of the child abuse questions were defined as having suffered from any child abuse. Information about child abuse was grouped into two non-overlapping categories: 'emotional and/or physical, not sexual' and 'sexual alone or in combination with emotional and/or physical'. Women could also indicate whether or not they had been abused during the past 12 months, and we defined this as recent abuse. All analyses of recent abuse refer to adult recent abuse, not child abuse. Past abuse refers to both child abuse and non-recent adult abuse.

\section{Perpetrators}

As part of the abuse questions, women were given the opportunity to reveal who committed the abuse: a stranger, family/relative or other known person (see online supplemental figure S1). The two latter categories were merged into known perpetrator. We categorised the responses about perpetrators into three groups: only known perpetrator, only unknown, and both known and unknown.

\section{Outcome variables: breast feeding}

The breastfeeding data are based on three questions about infant nutrition in the questionnaire completed 6 months postpartum. The questions asked about what type of milk (breast feeding or formula feeding) or other liquid the baby had been given in the first week of life and in monthly intervals up until and at the date of filling in the questionnaire (median 27 weeks/190 days). The women also reported the age (in months) of the infant at the time of introduction of semisolid or solid food. Full breast feeding was defined as predominant breast feeding without any formula or solids, but allowing water and vitamins. Any breast feeding included both full and partial breast feeding (ie, breast feeding with concomitant formula or solid foods given). The breastfeeding categories used in the present study are based on WHO definitions. ${ }^{32}$ In the present study, four dichotomous breastfeeding variables reflecting breastfeeding behaviour were used as outcome variables: (1) cessation of any breast feeding before 4 months, (2) cessation of full breast feeding before 4 months, (3) cessation of any breast feeding before 6 months and (4) cessation of full breast feeding before 6 months. We present descriptive data for all breastfeeding variables. As the main outcome, we present results only for the cessation of any breast feeding before 4 months, while results for the other breastfeeding variables are reported as text.

\section{Other variables}

Characteristics associated with any adult abuse in our study population have been examined previously ${ }^{30}$ and the following potential confounding variables were included based on this knowledge: being exposed to child abuse, civil status, maternal age, smoking and alcohol intake. We also included as potential confounding variables the following maternal characteristics and risk factors for early cessation of full or any breast feeding: maternal education, parity, body mass index (BMI), mode of delivery, preterm delivery, social support, prior depression and postpartum depression.

Information about maternal age, education, civil status, pre-pregnant weight and height (for calculating of BMI), parity, prior depression, social support, alcohol intake and smoking in pregnancy, was retrieved from the baseline questionnaire (week 18 in pregnancy). Information about postpartum depression was obtained from questionnaire 4 (6 months postpartum), whereas information about mode of delivery (caesarean section or vaginal delivery) and preterm delivery was retrieved from NMBR. The categorisation of age, education, civil status, parity, smoking and alcohol is shown in table 1 . BMI was calculated as weight in $\mathrm{kg} /$ (height) $^{2}$ (in $\mathrm{m}$ ) and dichotomised into $<25$ (underweight and normal weight) and $\geq 25$ (overweight and obese). Depression prior to current pregnancy was dichotomised into no or yes. Mode of delivery was categorised into vaginal birth or caesarean section. We defined preterm delivery as giving birth at $<37$ th week of gestation on the basis of ultrasound measurements. In the few cases, without ultrasound information $(<2 \%)$, gestational age was calculated from the first day of last menstrual period. Social support was defined as having anyone other than partner to ask for support, and was dichotomised into no or yes. Postpartum depression was identified and dichotomised based on four questions from the Edinburgh Postpartum Depression Scale and a cut-off score $>6$, which indicates a moderate level of postpartum depression symptoms. This variable has been described and examined previously in relation to adult exposure to abuse in MoBa. ${ }^{13}$

\section{Statistical analyses}

Descriptive statistics of the study population by exposure to any adult abuse are presented in table 1 . For testing differences between categories we used Pearson's $\chi^{2}$ test. We used binary logistic regression to examine the associations between adult abuse and early cessation of breast feeding. The reference group for all analyses of 
Table 1 Characteristics of the study population by any adult abuse in the Norwegian Mother and Child Cohort Study $(\mathrm{N}=53$ 934)

\begin{tabular}{|c|c|c|c|c|c|}
\hline & \multicolumn{2}{|l|}{ Total } & \multicolumn{2}{|c|}{ Any adult abuse } & \multirow[b]{2}{*}{ p Value* } \\
\hline & $\mathrm{n}$ & Per cent & $\mathbf{n}$ & Per cent & \\
\hline All & 53934 & 100 & 10442 & 19.4 & \\
\hline \multicolumn{6}{|l|}{ BF initiation } \\
\hline No & 618 & 1.1 & 147 & 23.8 & 0.005 \\
\hline Yes & 53316 & 98.9 & 10295 & 19.3 & \\
\hline \multicolumn{6}{|l|}{ Full BF for 4 months } \\
\hline No & 20991 & 38.9 & 4510 & 21.5 & $<0.001$ \\
\hline Yes & 32325 & 59.9 & 5785 & 17.9 & \\
\hline No BF initiation & 618 & & & & \\
\hline \multicolumn{6}{|l|}{ Full BF for 6 months } \\
\hline No & 45802 & 84.9 & 8896 & 19.4 & 0.102 \\
\hline Yes & 7514 & 13.9 & 1399 & 18.6 & \\
\hline No BF initiation & 618 & & & & \\
\hline \multicolumn{6}{|l|}{ Any BF for 4 months } \\
\hline No & 6539 & 12.1 & 1588 & 24.3 & $<0.001$ \\
\hline Yes & 46777 & 86.7 & 8707 & 18.6 & \\
\hline No BF initiation & & & 147 & & \\
\hline \multicolumn{6}{|l|}{ Any BF for 6 months } \\
\hline No & 10341 & 19.2 & 2445 & 23.6 & $<0.001$ \\
\hline Yes & 42945 & 79.7 & 7850 & 18.3 & \\
\hline No BF initiation & 618 & & 147 & & \\
\hline \multicolumn{6}{|l|}{ Age (years) } \\
\hline 14-19 & 693 & 1.3 & 101 & 14.6 & $<0.001$ \\
\hline $20-24$ & 6423 & 11.9 & 1162 & 18.1 & \\
\hline $25-29$ & 19628 & 36.4 & 3383 & 17.2 & \\
\hline $30-34$ & 21945 & 40.7 & 4390 & 20.0 & \\
\hline$\geq 35$ & 5245 & 9.7 & 1406 & 26.8 & \\
\hline \multicolumn{6}{|l|}{ Education } \\
\hline Primary (9 years) & 1195 & 2.2 & 332 & 27.8 & $<0.001$ \\
\hline Secondary (12 years) & 15902 & 29.5 & 3556 & 22.4 & \\
\hline Higher $\leq 4$ years & 31432 & 58.3 & 5218 & 16.6 & \\
\hline Higher $>4$ years & 3544 & 6.6 & 910 & 25.7 & \\
\hline Missing information & 1861 & 3.5 & 426 & 22.9 & \\
\hline \multicolumn{6}{|l|}{ Civil status } \\
\hline Married & 26572 & 49.3 & 4504 & 17.0 & $<0.001$ \\
\hline Cohabiting & 25543 & 47.4 & 5289 & 20.7 & \\
\hline Not married/cohabiting & 1523 & 2.8 & 578 & 38.0 & \\
\hline Missing information & 296 & 0.5 & 71 & 24.0 & \\
\hline \multicolumn{6}{|l|}{ Child abuse } \\
\hline No & 44064 & 81.7 & 7209 & 16.4 & $<0.001$ \\
\hline Yes & 9870 & 18.3 & 3233 & 32.8 & \\
\hline \multicolumn{6}{|l|}{ Parity } \\
\hline 0 & 27666 & 51.3 & 5155 & 18.6 & $<0.001$ \\
\hline+1 & 26268 & 48.7 & 5287 & 20.1 & \\
\hline \multicolumn{6}{|l|}{ Mode of delivery } \\
\hline Vaginal & 50296 & 93.3 & 9627 & 19.1 & $<0.001$ \\
\hline C-section & 3638 & 6.7 & 815 & 22.4 & \\
\hline \multicolumn{6}{|l|}{ Preterm delivery } \\
\hline No ( $\geq 37$ weeks) & 51258 & 95.0 & 9874 & 19.3 & 0.026 \\
\hline Yes (<37 weeks) & 2472 & 4.6 & 521 & 21.1 & \\
\hline Missing information & 204 & 0.4 & 47 & 23.0 & \\
\hline \multicolumn{6}{|l|}{ Smoking in pregnancy } \\
\hline No & 49100 & 91.0 & 8954 & 18.2 & $<0.001$ \\
\hline Yes & 4834 & 9.0 & 1488 & 30.8 & \\
\hline \multicolumn{6}{|l|}{ Alcohol in pregnancy } \\
\hline Never & 38931 & 72.2 & 7494 & 18.8 & $<0.001$ \\
\hline Sometimes & 7221 & 13.4 & 1628 & 22.5 & \\
\hline
\end{tabular}


Table 1 Continued

\begin{tabular}{|c|c|c|c|c|c|}
\hline & \multicolumn{2}{|l|}{ Total } & \multicolumn{2}{|c|}{ Any adult abuse } & \multirow[b]{2}{*}{ p Value* } \\
\hline & $\mathbf{n}$ & Per cent & $\mathbf{n}$ & Per cent & \\
\hline Daily & 47 & 0.1 & 19 & 40.4 & \\
\hline Missing information & 6705 & 12.4 & 1301 & 19.4 & \\
\hline \multicolumn{6}{|l|}{ BMI } \\
\hline$<25$ & 35389 & 66.5 & 6700 & 18.7 & $<0.001$ \\
\hline$\geq 25$ & 16552 & 30.7 & 3422 & 20.7 & \\
\hline Missing information & 1490 & 2.8 & 320 & 21.5 & \\
\hline \multicolumn{6}{|l|}{ Postpartum depression } \\
\hline No & 47349 & 87.8 & 8370 & 17.7 & $<0.001$ \\
\hline Yes & 5716 & 10.6 & 1897 & 33.2 & \\
\hline Missing information & 869 & 1.6 & 175 & 20.1 & \\
\hline
\end{tabular}

adult abuse was no adult abuse and the reference group for child abuse was no child abuse. Crude and adjusted ORs with 95\% CIs were presented and analysed for complete cases only. We included potential confounding variables based on previous knowledge of variables associated with either the exposure or the outcome. We identified potential confounders through directed acyclic graph (DAG) analysis. DAGs provide a method to identify potential confounders and decide which to adjust for. ${ }^{33}$ Many of the variables associated with both the exposure and the outcome in this study were intermediate variables rather than confounding variables. The minimal sufficient adjustment variables for estimating the total effect between adult abuse and breastfeeding behaviour were: maternal age, education, civil status and child abuse (see online supplemental figure S2), and these variables were included in all adjusted models. In addition, we evaluated the change in estimates when including intermediate variables: smoking, alcohol intake, parity, preterm delivery, social support, mode of delivery, BMI, preterm delivery and depression prior to pregnancy. Finally, we conducted a sensitivity analysis in which we stratified women according to postpartum depression to evaluate whether the association between adult abuse and breastfeeding behaviour was mediated primarily through postpartum depression. The data programme SPSS V.22 (SPSS Inc, IBM Company, Chicago, Illinois, USA) was used to conduct all analyses. A significance level of 0.05 was used.

\section{RESULTS}

The majority of the women in the study population initiated breast feeding (98.9\%). Nearly $14 \%$ of the infants were fully breast fed up to 6 months postpartum, while almost $80 \%$ were still breast fed (table 1). However, $12.1 \%$ of mothers ceased any breast feeding before 4 months and $38.9 \%$ ceased full breast feeding before 4 months. Overall, $19 \%$ of the 53934 women reported exposure to any adult abuse, and the prevalence of abuse was significantly higher in women who did not initiate breast feeding than in those who did. Likewise, any adult abuse was more prevalent in women who did not continue full or any breast feeding for 4 or 6 months. Exposure to abuse was more prevalent in women who were older, not married, had been exposed to child abuse, were parous, had caesarean delivery, smoked, reported drinking alcohol in pregnancy, were overweight or obese, and in women with postpartum depression.

Adult abuse was significantly associated with early cessation of breast feeding (table 2). Women exposed to any adult abuse had $25 \%$ increased adjusted ORs of cessation of any breast feeding before 4 months compared to their counterparts (table 2, model 1). When the other breastfeeding variables were used as the outcome, women who reported any adult abuse also had significantly increased odds of full breastfeeding cessation before 4 months and of any breastfeeding cessation before 6 months, respectively. However, no significant association was found between any adult abuse and full breastfeeding cessation before 6 months (data not shown).

In the analyses of singular or combined types of adult abuse, we found that women reporting emotional abuse only (adjusted OR 1.28, 95\% CI 1.18 to 1.39 ), emotional and physical abuse (adjusted OR 1.39, 95\% CI 1.18 to 1.62), emotional and sexual abuse (adjusted OR 1.27, 95\% CI 1.02 to 1.58 ) or those reporting all three types of abuse, that is, emotional, sexual and physical (adjusted OR 1.47, 95\% CI 1.23 to 1.76 ) were more likely to stop any breast feeding before 4 months than women without abuse (table 2, model 2 ).

Women reporting recent abuse (table 3) had $40 \%$ increased odds (adjusted OR 1.40, 95\% CI 1.24 to 1.58 ) of early cessation of any breast feeding compared to non-exposed women, while those reporting non-recent adult abuse had $21 \%$ increased odds of early breastfeeding cessation (adjusted OR 1.21, 95\% CI 1.12 to 1.30).

When abuse was grouped by type of perpetrator (table 4), exposure from 'known perpetrator only' was significantly associated with cessation of any breast 
Table 2 Logistic regression analyses of the association between types of adult abuse and cessation of any breast feeding (BF) before 4 months (cessation of any $B F<4$ months) $(\mathrm{N}=51$ 205)

\begin{tabular}{|c|c|c|c|c|c|c|c|c|}
\hline \multirow[b]{2}{*}{ Abuse category } & \multicolumn{4}{|c|}{ Cessation of any BF $<4$ months } & \multicolumn{2}{|c|}{ Crude } & \multicolumn{2}{|c|}{ Adjusted } \\
\hline & $\mathrm{n}$ & $(\%)$ & $\mathbf{n}$ & $(\%)$ & $\overline{\text { OR }}$ & $95 \% \mathrm{Cl}$ & $\overline{\text { OR }}$ & $95 \% \mathrm{Cl}$ \\
\hline \multicolumn{9}{|l|}{ Model 1} \\
\hline No adult abuse (reference) & 41396 & 80.8 & 4728 & 11.4 & 1.00 & & 1.00 & \\
\hline Any adult abuse & 9809 & 19.2 & 1511 & 15.4 & 1.41 & (1.33 to 1.50$)$ & 1.25 & (1.17 to 1.34$)$ \\
\hline \multicolumn{9}{|l|}{ Model 2 (abuse categories) } \\
\hline No adult abuse (reference) & 41396 & 80.8 & 4728 & 11.4 & 1.00 & & 1.00 & \\
\hline Physical only & 567 & 1.1 & 65 & 11.5 & 1.00 & (0.77 to 1.30$)$ & 0.96 & (0.73 to 1.25$)$ \\
\hline Sexual only & 976 & 1.9 & 107 & 11.0 & 0.96 & (0.78 to 1.17$)$ & 0.94 & (0.76 to 1.16$)$ \\
\hline Emotional only & 5464 & 10.7 & 843 & 15.4 & 1.42 & (1.31 to 1.53$)$ & 1.28 & (1.18 to 1.39$)$ \\
\hline Physical+emotional & 1149 & 2.2 & 210 & 18.3 & 1.73 & (1.49 to 2.02$)$ & 1.39 & (1.18 to 1.62$)$ \\
\hline Physical+sexual & 189 & 0.4 & 24 & 12.7 & 1.13 & (0.73 to 1.75$)$ & 0.95 & (0.61 to 1.47$)$ \\
\hline Sexual+emotional & 630 & 1.2 & 101 & 16.0 & 1.18 & (1.19 to 1.84$)$ & 1.27 & (1.02 to 1.58$)$ \\
\hline Sexual+physical+emotional & 827 & 1.6 & 161 & 19.3 & 1.86 & (1.56 to 2.21 ) & 1.47 & (1.23 to 1.76$)$ \\
\hline
\end{tabular}

The adjusted model included the following variables: maternal age, education, civil status and any child abuse. Analysed for complete cases.

feeding before 4 months (adjusted OR 1.28, 95\% CI 1.19 to 1.37$)$. The result for 'both known and unknown' perpetrators was significant in the crude model only, while exposure from 'unknown perpetrator only' was not associated with cessation of any breast feeding.

Compared with crude ORs, the adjusted ORs for the association between adult abuse and early breastfeeding cessation were attenuated to some degree, for example, from 1.41 to 1.25 in model 1 , table 2 . Of the four confounding variables, maternal education resulted in the largest change in the estimate. Additional adjustment for smoking, alcohol intake, parity, preterm delivery, social support, mode of delivery, BMI and depression prior to pregnancy did not substantially change in the OR of interest $(<10 \%)$, suggesting that the effect of adult abuse on early breastfeeding cessation was not mediated through these.

We have previously shown an association between abuse history and risk of postpartum depression, ${ }^{13}$ and we were particularly interested in examining postpartum depression as an intermediate variable in the analysis of adult abuse and early cessation of any breast feeding. The prevalence of breastfeeding cessation before 4 months was $19.3 \%$ among women with postpartum depression and $12.4 \%$ in those without postpartum depression. However, when stratifying women by postpartum depression, the association between any adult abuse and cessation of any breast feeding was evident and comparable in women with postpartum depression (adjusted OR 1.21, 95\% CI 1.12 to 1.30 ) and in those without (adjusted OR 1.23, 95\% CI 1.06 to 1.44). Accordingly, the association between exposure to abuse and early cessation of breast feeding cannot be explained by postpartum depression, rather by the abuse.

Exposure to child abuse was by itself significantly associated with any breastfeeding cessation before 4 months; the OR for any child abuse was 1.41 (95\% CI 1.32 to $1.50)$. When child abuse was categorised into "emotional and/or physical, not sexual' and 'sexual alone or in combination with emotional and/or physical', the association with breastfeeding cessation was OR 1.27 (95\% CI 1.17 to 1.37) for emotional and/or physical, and OR 1.66 (95\% CI 1.51 to 1.82 ) for sexual abuse. We had no available variables that could be considered confounders of child abuse. However, child abuse was still significantly associated with early breastfeeding cessation in the adjusted models, including adult abuse, maternal age, education and civil status with OR for any child abuse: 1.12 (95\% CI 1.05 to 1.20$)$. This association was stronger for sexual (sexual only or combined with other abuse) than for emotional and/or physical, not sexual, with OR $1.22(95 \%$ CI 1.11 to 1.65$)$ and OR 1.06 (95\% CI 0.98 to $1.15)$, respectively.

Table 3 Logistic regression analyses of the association between time of adult abuse (recent/not recent) and cessation of any breast feeding (BF) before 4 months (cessation of any $B F<4$ months) $(N=51014)$

\begin{tabular}{|c|c|c|c|c|c|c|c|c|}
\hline & \multicolumn{4}{|c|}{ Cessation of any BF $<4$ months } & \multicolumn{2}{|c|}{ Crude } & \multicolumn{2}{|c|}{ Adjusted } \\
\hline & $\mathbf{n}$ & (\%) & $\mathbf{n}$ & $(\%)$ & $\overline{\text { OR }}$ & $95 \% \mathrm{Cl}$ & OR & $95 \% \mathrm{Cl}$ \\
\hline No adult abuse (reference) & 41396 & 81.1 & 4728 & 11.4 & 1.00 & & 1.00 & \\
\hline Any adult, but not recent & 7495 & 14.7 & 1084 & 14.5 & 1.31 & (1.22 to 1.41$)$ & 1.21 & (1.12 to 1.30$)$ \\
\hline Any adult recent abuse & 2123 & 4.2 & 394 & 18.6 & 1.77 & (1.58 to 1.98$)$ & 1.40 & (1.24 to 1.58$)$ \\
\hline
\end{tabular}

The adjusted model included the following variables: maternal age, education, civil status and any child abuse. Analysed for complete cases. 
Table 4 Logistic regression analyses of the association between perpetrator of adult abuse and cessation of any breast feeding (BF) before 4 months (cessation of any $B F<4$ months) $(\mathrm{N}=51$ 101)

\begin{tabular}{|c|c|c|c|c|c|c|c|c|}
\hline \multirow[b]{2}{*}{ Perpetrator } & \multicolumn{4}{|c|}{ Cessation of any BF $<4$ months } & \multicolumn{2}{|c|}{ Crude } & \multicolumn{2}{|c|}{ Adjusted } \\
\hline & $\bar{n}$ & (\%) & $\mathbf{n}$ & $(\%)$ & OR & $95 \% \mathrm{Cl}$ & OR & $95 \% \mathrm{Cl}$ \\
\hline No adult abuse (reference) & 41396 & 81.0 & 4728 & 11.4 & 1.00 & & 1.00 & \\
\hline Known only & 7850 & 15.4 & 1232 & 15.7 & 1.44 & (1.35 to 1.55$)$ & 1.28 & (1.19 to 1.37$)$ \\
\hline Unknown only & 861 & 1.7 & 99 & 11.5 & 1.01 & (0.82 to 1.25$)$ & 1.09 & (0.88 to 1.35$)$ \\
\hline Known and unknown & 994 & 1.9 & 165 & 16.6 & 1.54 & (1.30 to 1.83$)$ & 1.18 & (0.99 to 1.41$)$ \\
\hline
\end{tabular}

The adjusted model included the following variables: maternal age, education, civil status and any child abuse. Analysed for complete cases.

\section{DISCUSSION}

The main finding in our study was that exposure to past and recent abuse was strongly associated with early cessation of any breast feeding. The strongest effect was seen for women exposed to three types of abuse (sexual, physical and emotional), with nearly $50 \%$ increased adjusted ORs of any breastfeeding cessation before 4 months compared to the non-exposed women. Recent abuse and exposure from known perpetrator resulted in nearly $40 \%$ and $30 \%$ increased risk of any breastfeeding cessation before 4 months, respectively. Women who reported a history of child abuse were more likely to stop breast feeding before 4 months than women who had not experienced child abuse. This was independent of later exposure to adult abuse. The theoretical causal pathway between adult abuse and breastfeeding behaviour is complex and it is challenging to disentangle which variables to use as confounders. We used a DAG approach and landed on including only maternal age, education, civil status and child abuse (see online supplemental figure S2). Of these, adjustment for maternal education resulted in the largest change of the estimate. Educational attainment has been shown to be strong indicator of socioeconomic differences in Norway. ${ }^{34-37}$ The DAG clarified how a number of the potential confounding variables were intermediate variables in the theoretical effect pathway and therefore, not true confounders. ${ }^{33}$ Furthermore, the sensitivity analyses showed that the estimated effect of abuse exposure on breastfeeding cessation was not primarily mediated through the intermediate variables. Interestingly, the association between adult abuse and breastfeeding cessation was evident both in women with and without postpartum depression.

\section{Strengths and limitations}

The major strengths of our study include the large sample size representing women from all regions of Norway, the prospective design and comprehensive information about singular and combined types of abuse, as well as extensive information on breast feeding. In addition, information about a wide range of potential confounding factors was available. The low participation rate in $\mathrm{MoBa}$ is a concern $(40.4 \%)$, with under-representation of women $<25$ years of age, smokers and those living alone. ${ }^{38}$ The potential selection bias in MoBa has been evaluated. Despite differences in prevalence estimates, associations between eight exposures and outcomes did not differ between MoBa participants and a representative sample from the general pregnant population, indicating that selection bias did not affect the associations. ${ }^{38}$ Retrospective reporting is a challenge, but difficult to avoid in this kind of study. The women's reporting of breast feeding 6 months postpartum could be subject to recall error. However, studies have found that maternal recall of breast feeding give accurate estimates shortly after delivery $^{39}$ and even 20 years after delivery, as described in a recent Norwegian study. ${ }^{40}$

\section{Comparison with other studies}

Previous studies have mainly investigated the associations between child sexual abuse, intimate partner violence (IPV) or pregnancy-related abuse and breast feeding. ${ }^{19-23} 41$ Our findings of abused women being significantly less likely to initiate breast feeding, and significantly more likely of early cessation of breast feeding, are in agreement with four other studies. ${ }^{19} 204243$ A study in 811 randomly selected women in five large primary health clinics in Brazil reported that severe physical IPV increased the risk of early breastfeeding cessation. ${ }^{19}$ A cross-sectional study comprising 1200 Chinese women showed that those who did not experience IPV during pregnancy were significantly more likely to initiate breast feeding than abused women. ${ }^{20} \mathrm{~A}$ review of 800 medical records in one family practice in the USA revealed an association between lack of breast feeding and physical and sexual abuse of mothers or their children. ${ }^{42}$ A longitudinal study in 296 adolescent females showed that participants who had experienced IPV ceased breast feeding earlier than their counterparts. ${ }^{43}$ Contrary to this, three studies found no differences in breast feeding between abused and non-abused women. ${ }^{21} \quad 22 \quad 44$ An Australian cluster randomised controlled trial involving 2621 women from 80 maternal and child health centres found that women exposed to IPV were less likely to initiate breast feeding than non-abused women, but rates of any breast feeding did not differ significantly between non-exposed women and those exposed to IPV when other factors such as maternal age and education were taken into account. ${ }^{21}$ Women in this study were older and had higher educational level than the general pregnant population, which are factors that may promote higher 
breastfeeding rates and underestimate abuse prevalence. Likewise, a large American population-based study $(\mathrm{n}=118579)$, found no significant association between recent IPV and any breastfeeding initiation or cessation during the first month postpartum. ${ }^{22}$ The results indicated that smoking and sociodemographic factors were more important predictors of breast feeding duration than abuse. ${ }^{22}$ Finally, no association was found between abuse and initiation and duration of breast feeding in a case-control study with 212 low-income women in two cities in the USA. ${ }^{44}$ The women were interviewed about past and recent domestic abuse, and classification of either breast feeding or formula feeding was determined by the type of food voucher the women received postpartum. The duration of breast feeding was assessed by the number of months the women received the vouchers. Important limitations of the study include the small sample size and possible sample bias. The authors discussed the possibility that women who had decided to breast feed were more likely to participate in the study than those who planned to bottle feed.

There are different reasons why results from the three studies differed from ours. One important factor is the differences in methodology and time frame of abuse as well as breast feeding. These also differ with regard to sample size and study design, which may influence prevalence rates of both abuse and breast feeding, respectively. Accordingly, one could argue that the low prevalence rates of IPV in two of the studies, that is, $6.3 \%{ }^{21}$ and $5.8 \%,{ }^{22}$ may be underestimated and influence the results. In comparison with other studies, our study has a large sample size, a prospective design and clearly defined exposure and outcome variables which corroborate the scientific evidence that past and recent abuse are negatively associated with breast feeding.

\section{Emotional abuse and breast feeding}

Few studies have examined emotional abuse and breast feeding, ${ }^{20} 23$ and to the best of our knowledge, no previous studies support the current finding of a significant association between emotional abuse as a singular or combined exposure and early cessation of any breast feeding. A cross-sectional study from Hong Kong $(n=1200)$ found that women who experienced emotional or physical abuse during pregnancy were more likely to be found in the artificial feeding group, than in the breast feeding or mixed feeding groups. ${ }^{20}$ In the current study, all abuse categories containing emotional abuse were significantly associated with cessation of any breast feeding in the adjusted models: emotional abuse only, emotional and sexual abuse, emotional and physical abuse, and emotional, physical and sexual abuse. This result is important and underpins that emotional abuse should be included when studying adverse health outcomes of past or recent abuse.

\section{Child abuse and breast feeding}

Child abuse was associated with both the exposure and the outcome in our study, and was modelled as a confounder. Furthermore, we found that child abuse was significantly associated with increased risk of any breastfeeding cessation before 4 months, independent of adult abuse. This association was stronger for child sexual abuse than for physical and/or emotional abuse only. The few existing previous studies that examined associations between child abuse and breast feeding have focused only on child sexual abuse and these have reported contradictory results. ${ }^{23} 4145$ The US study in 1220 nationally representative women, aforementioned, found that women with a history of self-reported child sexual abuse were twice as likely to initiate breast feeding as their non-abused counterparts, whereas breastfeeding duration did not differ significantly. ${ }^{23} \mathrm{~A}$ Canadian qualitative study found that the women's experiences of child sexual abuse affected their breastfeeding decisions, with the breastfeeding experience possibly resulting in re-traumatisation for some abused women and a healing effect on others. ${ }^{41}$ A literature review concluded that women with a history of child sexual abuse was more likely to express desire to and initiate breast feeding than their non-abused counterparts, but that both past and recent abuse could lead to breastfeeding cessation. ${ }^{45}$ A study from the USA in 1220 women from a nationally representative sample showed that childhood emotional and physical abuse was not significantly associated with breast feeding, whereas childhood sexual abuse was. ${ }^{23}$ The same study aimed to investigate a possible cumulative effect of abuse, but was unable to assess these effects due to few women reporting multiple types of abuse. Our results showed a strong independent effect of child abuse-particularly child sexual abuse-on breastfeeding cessation. This is an important finding and may indicate that sexual abuse early in life results in even worse adverse long-term effects than do other types of abuse.

\section{Public health implications}

During the past decades, several interventions to promote breast feeding in Norway have been implemented, and breastfeeding rates are higher in Norway than in most European countries. ${ }^{416}$ Baby-friendly hospitals, free antenatal care, free follow-up by community nurses and favourable maternity leave in Norway are societal priorities to enhance breastfeeding duration. Early maternal return to work can be a barrier both to initiating and duration of breast feeding. ${ }^{46}{ }^{47}$ Norway has a long parental leave which supports the possibility of breast feeding throughout the first year of life. ${ }^{48}$ Mean breastfeeding duration in Norway is about 10 months. Although, the majority of women in Norway breast feed for at least 6 months, a large decline in full breast feeding occurs between 3 and 4 months, and some women also discontinue any breast feeding within the first 6 months. ${ }^{15} 49$ An Australian longitudinal cohort 
study showed that women qualified for paid maternity leave had significantly reduced odds of reporting combined physical and emotional IPV the first year postpartum compared to non-working women. ${ }^{50}$ The current study showed that despite high breastfeeding rates and a favourable breastfeeding policy in Norway, past and recent abuse of women increased the prevalence of early breastfeeding cessation. These findings indicate that all women need to be screened for abuse during pregnancy because of its impact on maternal and child health. Recommendations urging caregivers to ask women about past and recent abuse have recently been implemented in the revised antenatal care guidelines in Norway. However, more research is needed on how antenatal care providers can recognise or ask about abuse, and which strategies to choose for support and breastfeeding assistance.

\section{CONCLUSION}

The current study shows that past and recent abuse of women is strongly associated with early cessation of breast feeding. Our results also underpin the need for emotional abuse to be included in studies of the adverse health effect of abuse. Given the convincing evidence of the beneficial effects of breast feeding both for the mother and the infant, it is crucial to promote high breastfeeding rates. Mothers with a history of past or recent abuse comprise a key group to target for extra support and breastfeeding assistance.

\section{Author affiliations}

${ }^{1}$ Faculty of Medicine, Department of Public Health and General Practice, Norwegian University of Science and Technology (NTNU), Trondheim, Norway ${ }^{2}$ Department of Physical Medicine and Rehabilitation, Aalesund Hospital, More and Romsdal Health Trust, Aalesund, Norway

${ }^{3}$ Faculty of Health Sciences, Department of Health, Nutrition and Management, Oslo and Akershus University College of Applied Sciences, Oslo, Norway ${ }^{4}$ Department of Exposure and Risk Assessment, Norwegian Institute of Public Health, Oslo, Norway

Acknowledgements The authors are grateful to all the families in Norway for participating in this ongoing cohort study.

Contributors MFS, $\mathrm{HG}$ and ML conceived the study, and all authors contributed to the study design. MFS and A-LB prepared the data and performed the statistical analyses. MFS drafted the manuscript. All authors contributed to the interpretation of the results and critically reviewed the manuscript. All authors read and approved the final manuscript.

Funding The Norwegian Mother and Child Cohort Study is supported by the Norwegian Ministry of Health and the Ministry of Education and Research, NIH/NIEHS (contract number N01-ES-75558), NIH/NINDS (grant number 1 U01 NS 047537-01 and grant number 2 U01 NS047537-06A1), and the Norwegian Research Council/FUGE (grant number 151918/S10). MFS was supported with a 3-year grant by the Norwegian Research Council (grant number 10334800) and was also supported with a 6-month scholarship by the More and Romsdal Health Trust, Aalesund Hospital, Aalesund, Norway. All authors are independent from funders.

\section{Competing interests None declared.}

Ethics approval The Norwegian Mother and Child Cohort Study was approved by the Regional Committee for Ethics in Medical Research (REK nr S-97045 /S-95113) and the Data Inspectorate in Norway.
Provenance and peer review Not commissioned; externally peer reviewed.

Data sharing statement No additional data are available.

Open Access This is an Open Access article distributed in accordance with the Creative Commons Attribution Non Commercial (CC BY-NC 4.0) license, which permits others to distribute, remix, adapt, build upon this work noncommercially, and license their derivative works on different terms, provided the original work is properly cited and the use is non-commercial. See: http:// creativecommons.org/licenses/by-nc/4.0/

\section{REFERENCES}

1. WHO. Evidence of the long term effects of breastfeeding: systematic reviews and meta-analyses. WHO/NHD/01082007. Geneva: World Health Organization, 2007.

2. Ip S, Chung M, Raman G, et al. Breastfeeding and Maternal and Infant Health Outcomes in Developed Countries. Evidence Report/ Technology Assessment. 2007:1-186.

3. Natland ST, Nilsen TI, Midthjell K, et al. Lactation and cardiovascular risk factors in mothers in a population-based study: the HUNT-study. Int Breastfeed J 2012;7:8.

4. Hörnell A, Lagström H, Lande B, et al. Breastfeeding, introduction of other foods and effects on health: a systematic literature review for the 5th Nordic Nutrition Recommendations. Food Nutr Res 2013;57:10.3402/fnr.v57i0.20823. Published Online 12 Apr 2013. doi:10.3402/fnr.v57i0.20823.

5. Dermer A. Breastfeeding and Women's Health. J Womens Health (Larchmt) 1998;7:427.

6. WHO. The optimal duration of exclusive breastfeeding. Report of an expert consultation, Geneva, Switzerland, 28-30 March 2001.

7. Kramer MS, Kakuma R. Optimal duration of exclusive breastfeeding. Cochrane Database Syst Rev 2012;8:CD003517.

8. WHO. Global strategy for infant and young child feeding. 2003. http://www.who.int/nutrition/publications/infantfeeding/9241562218/ en/

9. Norwegian Directorate of Health. Recommendations for infant feeding during the first year of life [in Norwegian]. https://helsedirektoratet.no/ folkehelse/kosthold-og-ernering/spedbarnsernering-mat-og-drikke-detforste-aret http://helsedirektoratet.no/lists/publikasjoner/anbefalingerfor-spedbarnsernering.pdf

10. WHO. WHO report highlights violence against women as a 'global health problem of epidemic proportions'. New clinical and policy guidelines launched to guide health sector response. WHO in partnership with the London School of Hygiene \& Tropical Medicine and the South African Medical Research Council. WHO media release. WHO, the London School of Hygiene \& Tropical Medicine and the South African Medical Research Council, 2013. http://www. who.int/mediacentre/news/releases/2013/violence_against_women_ 20130620/en/index.html

11. WHO. Global and regional estimates of violence against women: prevalence and health effects of intimate partner violence and non-partner sexual violence. Department of Reproductive Health and Research, London School of Hygiene and Tropical Medicine, South African Medical Research Council, 2013.

12. García-Moreno $\mathrm{C}$, Stöckl H. Protection of sexual and reproductive health rights: addressing violence against women. Int J Gynaecol Obstet 2009;106:144-7.

13. Sørbø MF, Grimstad H, Bjørngaard JH, et al. Adult physical, sexual, and emotional abuse and postpartum depression, a population based, prospective study of 53,065 women in the Norwegian Mother and Child Cohort Study. BMC Pregnancy Childbirth 2014;14:316.

14. Pallitto CC, García-Moreno C, Jansen HAFM, et al. Intimate partner violence, abortion, and unintended pregnancy: results from the WHO Multi-country Study on Women's Health and Domestic Violence. Int $J$ Gynecol Obstet 2013;120:3-9.

15. Kristiansen AL, Lande B, Øverby NC, et al. Factors associated with exclusive breast-feeding and breast-feeding in Norway. Public Health Nutr 2010;13:2087-96.

16. Callen J, Pinelli J. Incidence and duration of breastfeeding for term infants in Canada, United States, Europe, and Australia: a literature review. Birth 2004;31:285-92.

17. Lande B, Andersen LF, Bærug A, et al. Infant feeding practices and associated factors in the first six months of life: the Norwegian Infant Nutrition Survey. Acta Pædiatr 2003;92:152-61.

18. Winkvist A, Brantsæter AL, Brandhagen $M$, et al. Maternal body mass index and gestational weight gain are associated with initiation and duration of breastfeeding among Norwegian mothers. J Nutr 2015;145:1263-70. 
19. Moraes CL, de Oliveira AS, Reichenheim ME, et al. Severe physical violence between intimate partners during pregnancy: a risk factor for early cessation of exclusive breast-feeding. Public Health Nutr 2011;14:2148-55.

20. Lau Y, Chan KS. Influence of intimate partner violence during pregnancy and early postpartum depressive symptoms on breastfeeding among Chinese women in Hong Kong. J Midwifery Womens Health 2007;52:e15-20.

21. James JP, Taft A, Amir LH, et al. Does intimate partner violence impact on women's initiation and duration of breastfeeding? Breastfeed Rev 2014;22:11-19.

22. Silverman JG, Decker MR, Reed E, et al. Intimate partner violence around the time of pregnancy: association with breastfeeding behavior. J Womens Health (Larchmt) 2006;15:934-40.

23. Prentice JC, Lu MC, Lange L, et al. The association between reported childhood sexual abuse and breastfeeding initiation. J Hum Lact 2002;18:219-26.

24. Magnus $\mathrm{P}$, Irgens LM, Haug $\mathrm{K}$, et al. Cohort profile: the Norwegian Mother and Child Cohort Study (MoBa). Int $J$ Epidemiol 2006;35:1146-50.

25. von Elm E, Altman DG, Egger M, et al. The strengthening of the Reporting of Observational Studies in Epidemiology (STROE) statement: guidelines for reporting observational studies. J Clin Epidemiol 2008;61:344-9.

26. Swahnberg IM, Wijma B. The NorVold Abuse Questionnaire (NorAQ): validation of new measures of emotional, physical, and sexual abuse, and abuse in the health care system among women. Eur J Public Health 2003;13:361-6.

27. Rabin RF, Jennings JM, Campbell JC, et al. Intimate partner violence screening tools: a systematic review. Am J Prev Med 2009;36:439-45.e4.

28. Stenson K, Heimer G, Lundh C, et al. Lifetime prevalence of sexual abuse in a Swedish pregnant population. Acta Obstet Gynecol Scand 2003:82:529-36.

29. Johnson JK, Haider F, Ellis K, et al. The prevalence of domestic violence in pregnant women. BJOG 2003;110:272-5

30. Sørbø MF, Grimstad H, Bjørngaard JH, et al. Prevalence of sexual, physical and emotional abuse in the Norwegian mother and child cohort study. BMC Public Health 2013;13:186.

31. Lukasse M, Schei B, Vangen S, et al. Childhood abuse and common complaints in pregnancy. Birth 2009;36:190-9.

32. WHO Indicators for assessing infant and young child feeding practices. Part 1: definitons. Geneva: WHO, 2008. http://www. whqlibdoc.who.int/publications/2008/9789241596664_eng.pdf

33. Greenland S, Pearl J, Robins JM. Causal diagrams for epidemiologic research. Epidemiology 1999;10:37-48.

34. Nilsen SM, Bjørngaard JH, Ernstsen L, et al. Education-based health inequalities in 18,000 Norwegian couples: the Nord-Trøndelag Health Study (HUNT). BMC Public Health 2012;12:998.

35. Nilsen SM, Krokstad S, Holmen TL, et al. Adolescents health-related dietary patterns by parental socio-economic position, the Nord-Trøndelag Health Study (HUNT). Eur J Public Health 2010;20:299-305.

36. Petersen CB, Mortensen LH, Morgen CS, et al. Socio-economic inequality in preterm birth: a comparative study of the Nordic countries from 1981 to 2000. Paediatr Perinat Epidemiol 2009;23:66-75.

37. Nilsen SM, Bjørngaard JH, Erntsen L, et al. Education-based health inequalities in 18,000 Norwegian couples: the Nor-Trøndelag Health Study (HUNT). BMC Public Health 2012;12:998.

38. Nilsen RM, Vollset SE, Gjessing HK, et al. Self-selection and bias in a large prospective pregnancy cohort in Norway. Paediatr Perinat Epidemiol 2009;23:597-608.

39. Cupul-Uicab LA, Gladen BC, Hernández-Ávila M, et al. Reliability of reported breastfeeding duration among reproductive-aged women from Mexico. Matern Child Nutr 2009;5:125-37.

40. Natland S, Andersen L, Nilsen T, et al. Maternal recall of breastfeeding duration twenty years after delivery. BMC Med Res Methodol 2012;12:179.

41. Wood K, Van Esterik P. Infant feeding experiences of women who were sexually abused in childhood. Can Fam Physician 2010;56: e136-41.

42. Acheson L. Family violence and breast-feeding. Arch Fam Med 1995;4:650-2.

43. Sipsma HL, Magriples U, Divney A, et al. Breastfeeding behavior among adolescents: initiation, duration, and exclusivity. J Adolesc Health 2013;53:394-400.

44. Bullock LF, Libbus MK, Sable MR. Battering and breastfeeding in a WIC population. Can J Nurs Res 2001;32:43-56.

45. Kendall-Tackett KA. Violence against women and the perinatal period: the impact of lifetime violence and abuse on pregnancy, postpartum, and breastfeeding. Trauma Violence Abuse 2007:8:344-53

46. Chuang $\mathrm{CH}$, Chang $\mathrm{PJ}$, Chen $\mathrm{YC}$, et al. Maternal return to work and breastfeeding: a population-based cohort study. Int J Nurs Stud 2010;47:461-74.

47. Mandal B, Roe BE, Fein SB. The differential effects of full-time and part-time work status on breastfeeding. Health Policy 2010;97:79-86.

48. NAV (Norwegian Social Welfare System). Parental benefit in Norway Information in English. https://www.nav.no/en/Home/Benefits+and +services/Relatert+informasjon/Parental+benefit.353588.cms (accessed 08 May 2015).

49. Häggkvist AP, Brantsæter AL, Grjibovski AM, et al. Prevalence of breast-feeding in the Norwegian Mothe and Child Cohort Study and health service-related correlates of cessation of full breast-feeding. Public Health Nutr 2010;13:2076-86.

50. Gartland D, Hemphill SA, Hegarty $\mathrm{K}$, et al. Intimate partner violence during pregnancy and the first year postpartum in an Australian pregnancy cohort study. Matern Child Health $J$ 2011;15:570-8. 